$16^{\text {th }}$ International Congress of Metrology, 11005 (2013)

DOI: $10.1051 /$ metrology/201311005

(C) Owned by the authors, published by EDP Sciences, 2013

\title{
Mesure d'énergie électrique dans le domaine ferroviaire
}

\author{
Mohamed Agazar ${ }^{1, \mathrm{a}}$, André Poletaeff ${ }^{1}$, Dominique Fortune ${ }^{1}$, Antoine Feuillatre ${ }^{2}$ et Isabelle Blanc ${ }^{1}$. \\ ${ }^{1}$ LNE (Laboratoire National de métrologie et d'Essais), département métrologie électrique basses fréquences, 29 Avenue \\ Roger Hennequin 78197 Trappes, France \\ ${ }^{2}$ École spéciale de mécanique et d'électricité, 51 Boulevard de Brandebourg, 94200 Ivry-sur-Seine
}

\begin{abstract}
The directive of European railway interoperability promotes the movements of trains on any rail network without any difference, including networks in different states. The energy consumed must be measured for each train in any state. In Europe, the electrification of train can be done by either a high DC voltage or a high $\mathrm{AC}$ voltage at frequencies of $50 \mathrm{~Hz}$ and $16 \mathrm{~Hz} 2 / 3$. LNE has a good expertise in DC high voltage measurements and in $\mathrm{AC}$ high voltage measurements at frequency of $50 \mathrm{~Hz}$ but not for the frequency of $16 \mathrm{~Hz} 2 / 3$. This article presents, then, an overview of the work that has been done or that is actually in progress in LNE to ensure the traceability of measurements at frequency of $16 \mathrm{~Hz} 2 / 3$, frequency of railway electrification in Germany, in Switzerland and in Austria. The work mainly concerns the generation and the measurements of a high voltage up to $30 \mathrm{kV}$, the current measurements up to $250 \mathrm{~A}$, the active and the reactive power measurements and the low voltage measurements up to $1000 \mathrm{~V}$.
\end{abstract}

\section{Introduction}

Dans le contexte de libéralisation du marché du ferroviaire, les différents exploitants sont incités à posséder des équipements de mesure d'énergie sur les motrices des trains. Ces compteurs d'énergie doivent permettre de donner une image exacte de la consommation d'énergie de chaque motrice. En fonction de la situation géographique du train, l'électrification ferroviaire peut se faire en tension continue ou en tensions alternatives de fréquences $50 \mathrm{~Hz}$ ou $16 \mathrm{~Hz} 2 / 3$. Cette dernière concerne essentiellement l'énergie d'électrification en Suisse, en Allemagne et en Autriche. Le LNE possède une expertise dans les domaines de la haute tension, du fort courant et du comptage d'énergie, mais ne possédait pas d'expérience dès lors que ces grandeurs concernent la fréquence $16 \mathrm{~Hz}$ $2 / 3$. Le LNE a donc entrepris un ensemble de travaux, présenté dans cet article, afin de garantir la traçabilité des mesures ferroviaires au SI pour cette fréquence.

\section{Système de mesure d'énergie ferroviaire}

Le système de mesure d'énergie «Energy Measurement Function»(EMF) incorporé dans les motrices des trains, dont le principe est présenté dans la figure 1, permet de mesurer la quantité d'énergie active ou réactive consommée par une unité de traction. L'EMF contient essentiellement trois fonctions de mesure, une fonction de

\footnotetext{
${ }^{\mathrm{a}}$ Mohamed Agazar: mohamed.agazar@lne.fr
}

mesure de courant «Current Measurements function» $(\mathrm{CMF})$, une fonction de mesure de tension «Voltage Measurement Function» (VMF) et une fonction de mesure de puissance «Energy Calculation Function» (ECF). Les classes de précision des instruments de mesure sont définies dans la norme EN 50463-2.

Un système de traitement «Data Handling System» permet de stocker les données et de produire les informations nécessaires à la facturation de l'énergie consommée.

En fonction de la localisation géographique du train, différents systèmes d'alimentation sont utilisés; $25 \mathrm{kV}$ à $50 \mathrm{~Hz}, 15 \mathrm{kV}$ à $16 \mathrm{~Hz} 2 / 3$ et de $600 \mathrm{~V}$ à $3 \mathrm{kV}$ en courant continu. Les courants peuvent atteindre quelques centaines d'ampères en alternatif et quelques milliers d'ampères en continu. Pour les mesurer, des colonnes hautes tensions et des shunts forts courants sont donc incorporés dans l'EMF. Ils sont associés à des éléments de mesure et de traitement basses tensions.

\section{Traçabilité au SI des mesures des hautes tensions en $16 \mathrm{~Hz} 2 / 3$}

\subsection{Génération de la haute tension}

Un système pour générer une tension à la fréquence de $16 \mathrm{~Hz} 2 / 3$ a été développé au LNE. Le principe consiste à 


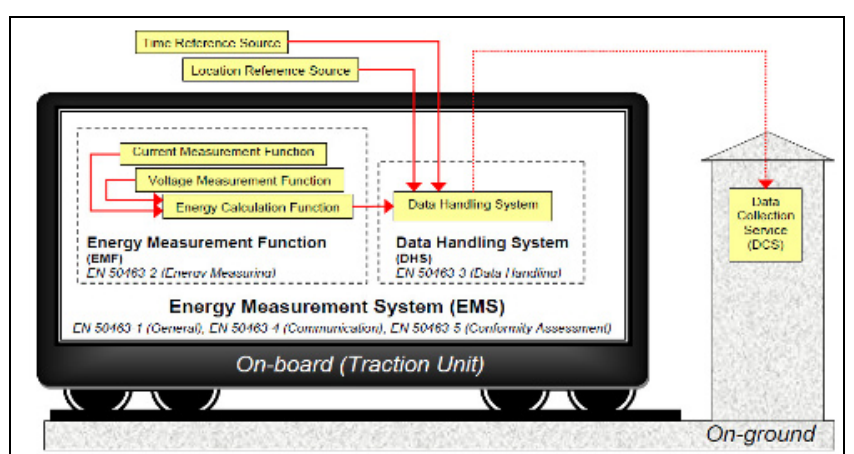

Figure 1. Système de mesure d'énergie ferroviaire.

utiliser un transformateur élévateur dont l'enroulement secondaire est alimenté par un amplificateur de puissance capable de fournir une puissance de $5 \mathrm{~kW}$. Ce dernier est piloté par un générateur basse fréquence pouvant fournir des tensions de l'ordre de quelques volts à la fréquence de $16 \mathrm{~Hz} 2 / 3$. Aujourd'hui le LNE est capable de générer une haute tension de $30 \mathrm{kV}$ à cette fréquence avec des instabilités inférieures à $100 \mu \mathrm{V} / \mathrm{V}$ et avec un taux de distorsion global inférieur à $0,1 \%$.

\subsection{Etalonnage des kilovoltmètres}

L'étalonnage des éléments du VMF se fait par comparaison directe à un kilovoltmètre (un kilovoltmètre est un diviseur haute tension associé à un voltmètre). La traçabilité de ce dernier se fait par comparaison directe à un kilovolmètre étalon suivant le schéma de la figure 2 .

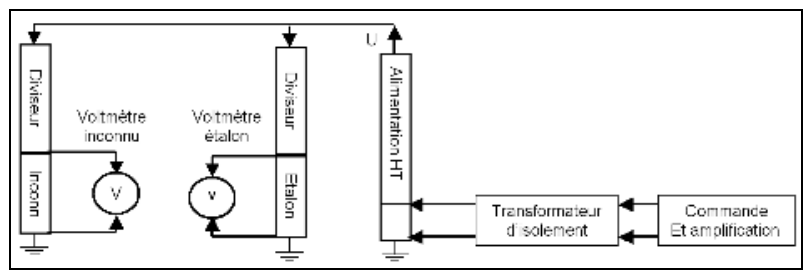

Figure 2. Etalonnage d'un kilovoltmètre.

La tension est appliquée sur la partie haute tension du diviseur inconnu et sur la partie haute tension du diviseur étalon. Les deux diviseurs sont associés à leur voltmètre respectif. Le coefficient de conversion $\mathrm{K}_{\mathrm{x}} \mathrm{du}$ kilovoltmètre en étalonnage est donné par la formule (1)

$$
K_{x}=\frac{L_{e} \cdot K_{e}}{L_{x}}
$$

Avec $\mathrm{L}_{\mathrm{e}}$ la lecture du voltmètre étalon, $\mathrm{L}_{\mathrm{x}}$ la lecture $\mathrm{du}$ voltmètre inconnu et $\mathrm{K}_{\mathrm{e}}$ le coefficient de conversion du diviseur étalon.

Le diviseur de référence a été constitué d'une capacité haute tension de $100 \mathrm{pF} / 100 \mathrm{kV}$ associé à une capacité de pied basse tension de $100 \mathrm{nF}$. La caractérisation de ce diviseur en température, en tension et en fréquence a été effectuée. Les résultats de la caractérisation de ce diviseur en fréquence sont présentés dans la figure 3.

Les incertitudes de mesures courantes sont inférieures à $5.10^{-4} \cdot \mathrm{K}_{\mathrm{x}}(\mathrm{k}=1)$.

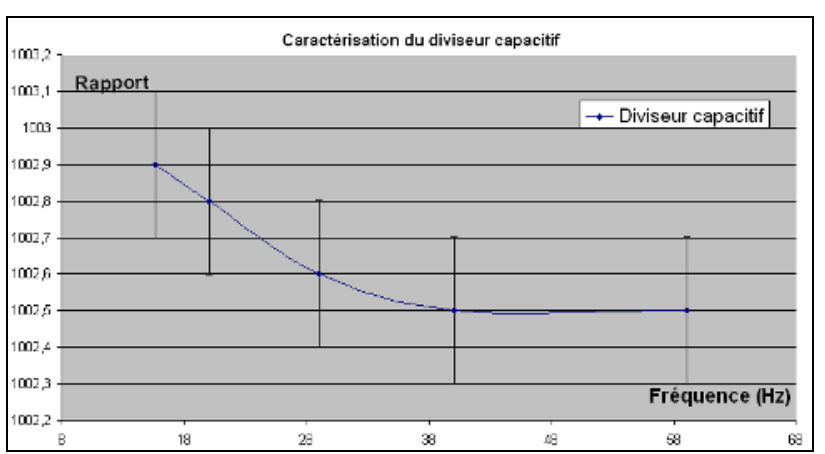

Figure 3. Réponse en fréquence du diviseur capacitif.

\subsection{Etalonnage des transformateurs de tension}

Un banc de caractérisation de transformateurs de tension à la fréquence de $16 \mathrm{~Hz} 2 / 3$ est en cours de réalisation au LNE. Son fonctionnement est basé sur un échantillonnage et une conversion analogique numérique des signaux. Le principe de mesure est présenté dans la figure (4).

Le traitement des échantillons est fait par transformation de Fourier complexe permettant d'accéder à l'amplitude et à la phase de la fréquence fondamentale ainsi qu'à l'amplitude et à la phase des harmoniques.

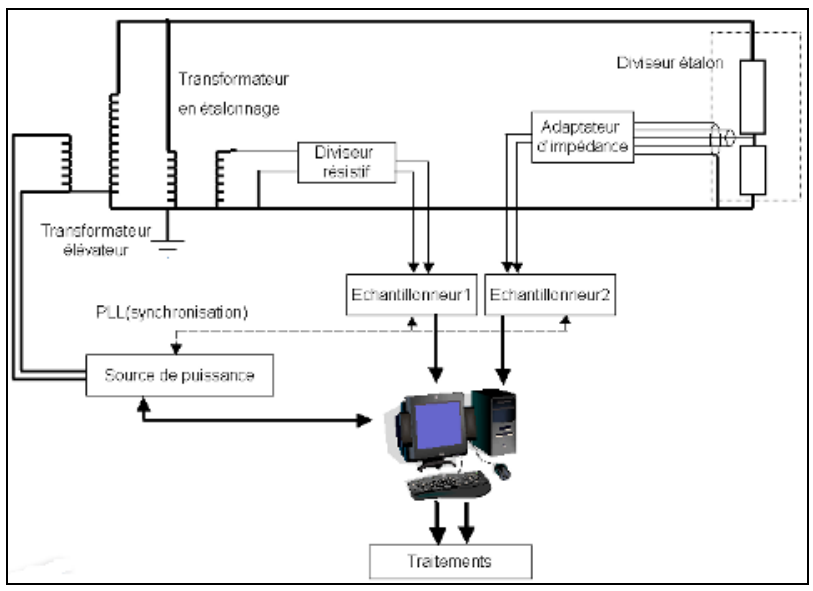

Figure 4. Banc d'étalonnage des transformateurs de tension.

L'erreur de rapport et le déphasage entre la tension primaire et la tension secondaire du transformateur sont mesurées.

Le rapport entre la tension primaire et la tension secondaire d'un transformateur de tension est donné par la formule (2):

$$
K=\frac{U_{P}}{U_{S}}=K_{n} /(1+\varepsilon)
$$

avec $\mathrm{U}_{\mathrm{P}}, \mathrm{U}_{\mathrm{s}}, \mathrm{K}_{\mathrm{n}}$ et $\varepsilon$ sont respectivement la tension primaire, la tension secondaire, le rapport de transformation nominal et l'erreur de rapport.

L'erreur de rapport du transformateur en étalonnage $\varepsilon$ est donnée en fonction des éléments du montage par la formule (3).

$$
\varepsilon_{x}=\frac{K_{n} \cdot K_{D} \cdot V_{X}}{K_{e} \cdot K_{A} \cdot V_{E}}-1
$$


$\mathrm{K}_{\mathrm{n}}$ et $\mathrm{K}_{\mathrm{e}}$ sont respectivement le rapport nominal du transformateur en étalonnage et le rapport réel du diviseur étalon. $\mathrm{K}_{\mathrm{A}}$ et $\mathrm{K}_{\mathrm{D}}$ sont respectivement le rapport de l'étage adaptateur et le rapport du diviseur résistif. $V_{E}$ et $V_{X}$ sont respectivement la tension mesurée par l'échantillonneur 2 et la tension mesurée par l'échantillonneur 1.

Le déphasage du transformateur en étalonnage $\varphi_{\mathrm{x}}$ est donné par la formule (4):

$$
\varphi_{x}=\varphi_{x-e}+\varphi_{e}+\varphi_{D}-\varphi_{A}
$$

$\varphi_{\mathrm{e}}, \varphi_{\mathrm{D}}, \varphi_{\mathrm{A}}$ et $\varphi_{\mathrm{x}-\mathrm{e}}$ sont respectivement le déphasage $\mathrm{du}$ diviseur étalon, le déphasage du diviseur résistif, le déphasage de l'étage adaptateur et le déphasage mesuré entre les deux échantillonneurs 1 et 2.

L'étalon est un diviseur résistif, ses caractéristiques en fréquence et en température ont été déterminées dans la gamme de fréquences du DC à $1000 \mathrm{~Hz}$.

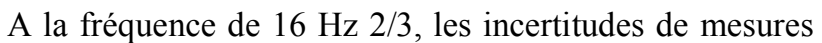
envisagées par cette méthode sont meilleures que 100 $\mu \mathrm{V} / \mathrm{V}$ pour l'erreur de rapport et meilleures que $100 \mu \mathrm{rad}$ pour le déphasage.

\section{Traçabilité au SI des mesures des courants en $16 \mathrm{~Hz}$ 2/3}

A la fréquence de $16 \mathrm{~Hz} 2 / 3$, l'usage de transformateurs de courant n'est pas adéquat à cause de la saturation des circuits magnétiques. L'utilisation de shunts et de résistances pour la mesure des courants est plus adéquate. La traçabilité des shunts au SI se fait par comparaison à un shunt étalon au moyen d'un banc de comparaison de résistances, figure 5, dont le principe repose sur une technique d'échantillonnage et un traitement numérique par une méthode d'ajustement par les moindres carrés.

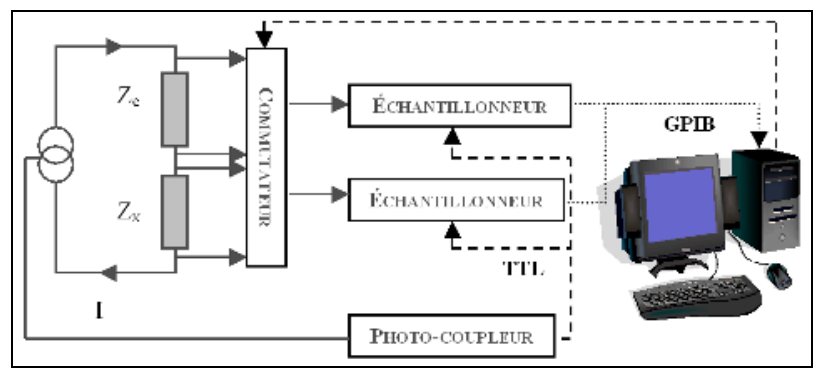

Figure 5. Banc de comparaison de résistances.

Un même courant alternatif sinusoïdal I parcourt le shunt étalon $Z_{e}$ et le shunt en étalonnage $Z_{x}$. Les tensions développées aux bornes des deux shunts sont alors échantillonnées puis traitées afin de déterminer la valeur de la résistance série $R_{x}$ et son déphasage $\varphi_{x}$. L'impédance complexe $Z_{x}$ peut alors s'écrire :

$$
Z_{x}=R_{x}\left(1+j \tan \left(\varphi_{x}\right)\right)
$$

Les mesures couvrent le domaine de $1 \mathrm{~m} \Omega$ à $5 \mathrm{M} \Omega$ pour des fréquences allant jusqu'à $20 \mathrm{kHz}$ et des courants pouvant atteindre $250 \mathrm{~A}$. Au courant maximum, les incertitudes de mesures sont de l'ordre de $1.10^{-4} \cdot \mathrm{R}(\mathrm{k}=1)$ pour la valeur de la résistance et quelques dizaines de $\mu$ rad pour le déphasage.

\section{Traçabilité au SI des mesures de puissances en $16 \mathrm{~Hz}$ 2/3.}

Le LNE dispose d'un banc primaire pour l'étalonnage des wattmètres. L'étalonnage de ces derniers se fait au moyen du wattmètre à échantillonnage numérique, le principe d'étalonnage est présenté dans la figure 6 .

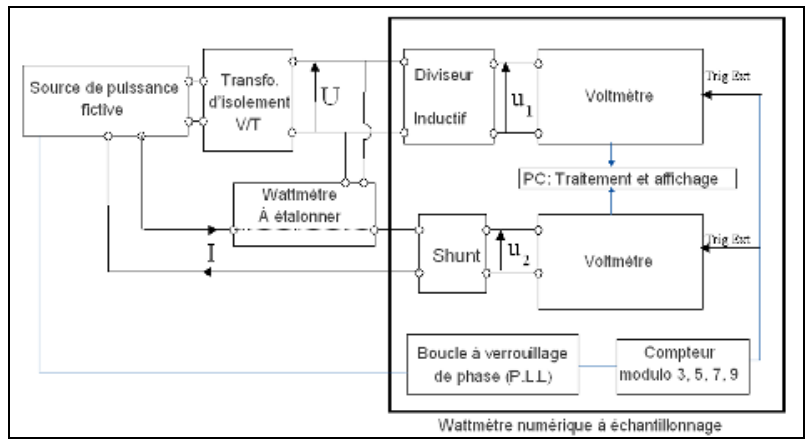

Figure 6. Banc d'étalonnage des Wattmètres.

Les signaux de tension $U$ et de courant I délivrés par la source de puissance fictive sont appliqués à un diviseur inductif et à un shunt respectivement. Les tensions $u_{1}(t)$ à la sortie $\mathrm{du}$ diviseur inductif et $\mathrm{u}_{2}(\mathrm{t})$ développée aux bornes du shunt sont mesurées par deux voltmètres sur le calibre $1 \mathrm{~V}$ en mode échantillonnage (mode DCV). Les échantillons ainsi obtenus sont traités par Transformée de Fourier Discrète (TFD) donnant accès, pour les différentes composantes spectrales, aux valeurs efficace $\mathrm{U}$ de la tension et I du courant ainsi qu'au déphasage $\phi$ entre la tension et le courant et permettant le calcul de la puissance active $\mathrm{P}$. Ce banc a été conçu pour fonctionner sur un intervalle de fréquence allant de $50 \mathrm{~Hz}$ à $400 \mathrm{~Hz}$. Les incertitudes de mesure sont inférieures à $30 \mu \mathrm{W} / \mathrm{VA}(\mathrm{k}=1)$.

L'extension des mesures à la fréquence de $16 \mathrm{~Hz} 2 / 3$ est en cours de réalisation. Les caractéristiques des diviseurs inductifs étant mal appropriées en basses fréquences à cause de la saturation du circuit magnétique, il est préférable d'utiliser des diviseurs résistifs convenablement étudiés et compensés pour présenter un déphasage négligeable ou connu avec des incertitudes inférieures à $5 \mu \mathrm{rad}$.

Les incertitudes en $16 \mathrm{~Hz} 2 / 3$ peuvent être meilleures que $200 \mu \mathrm{rad} / \mathrm{VA}(\mathrm{k}=1)$ pour une tension de $1000 \mathrm{~V}$ et un courant de $250 \mathrm{~A}$.

\section{Traçabilité des mesures en basses tensions en $16 \mathrm{~Hz}$ 2/3}

Les voltmètres peuvent être utilisés par exemple pour mesurer la tension développée aux bornes d'un shunt afin d'en déduire le courant ou encore mesurer la tension à la sortie d'un diviseur haute tension afin d'en déduire la valeur de la haute tension. Il faut savoir que leur 
traçabilité est définie en régime continu. Les paramètres servant à caractériser les signaux alternatifs sont donc raccordés à ces unités par les convertisseurs thermiques. En effet, La transposition thermique alternatif-continu est actuellement le moyen le plus précis d'effectuer ce raccordement pour la valeur efficace des signaux. Le principe (voir figure 7) consiste donc à appliquer successivement le signal alternatif à mesurer puis un signal continu ajustable à une résistance chauffante dont l'échauffement est repéré au moyen d'un ou plusieurs thermocouples fixés sur cette résistance. Lorsque la tension délivrée par les thermocouples en réponse au signal alternatif et au signal continu est identique, on conclut que la valeur efficace du signal alternatif est égale à la valeur du signal continu (servant de référence) à l'erreur près (ou écart de transposition) du convertisseur thermique.

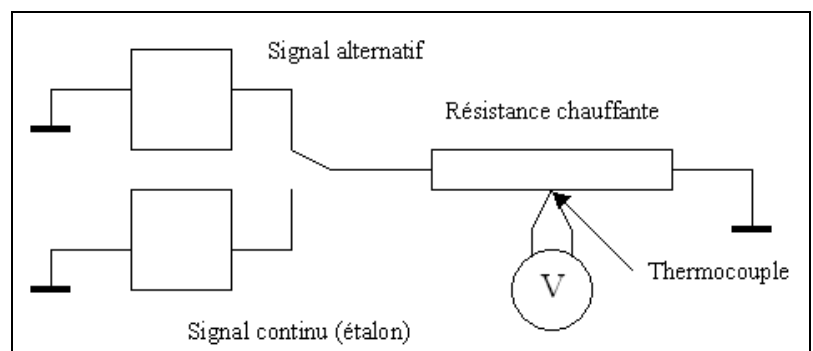

Figure 7. Principe de la transposition thermique.

Les références primaires du LNE sont constituées par des convertisseurs dont la constitution et la géométrie permettent le calcul de leur écart de transposition. Les autres convertisseurs (étalons secondaires) sont raccordés à ceux-ci au moyen de bancs d'étalonnage automatiques. L'ensemble des convertisseurs (référence primaire + références secondaires) couvrent déjà un domaine contenant la fréquence de $16 \mathrm{~Hz} 2 / 3$ pour des tensions comprises entre $0,5 \mathrm{~V}$ et $1000 \mathrm{~V}$.

\section{Conclusion}

Les travaux réalisés ou en cours de réalisation au LNE offrent maintenant la possibilité d'effectuer des mesures à la fréquence de $16 \mathrm{~Hz} \mathrm{2/3}$ pour des tensions allant jusqu'à $30 \mathrm{kV}$ et pour des courants allant jusqu'à $250 \mathrm{~A}$. Combiné avec son savoir faire en tension et courant continus et en tension et courant alternatifs aux fréquences de $50 \mathrm{~Hz}$ et $60 \mathrm{~Hz}$, le LNE est en mesure de répondre à toutes les demandes couvrant le domaine ferroviaire.

\section{Références}

1. Patrick Espel, Andre Poletaeff; « Nouvelles possibilités d'étalonnage en puissance électrique au LNE » Revue française de métrologie, 26, 2011-2.

2. Andre Poletaeff; «Automated Comparator for Accurate AC-DC Difference Measurements at the BNMLCIE» IEEE trans., Vol. 48, $\mathrm{n}^{\circ}$ 2, pp: 412-414, April 1999.
3. Jérôme Manceau; «application des méthodes d'échantillonnage aux mesures des déphasages pour des fréquences de $20 \mathrm{~Hz}$ à $20 \mathrm{kHz}$ » Revue française de métrologie, 13, 2008-1 